\title{
Combined posterolateral knee reconstruction: ACL-based injuries perform better compared to PCL-based injuries
}

\author{
Patricia M. Lutz ${ }^{1}$ - Michael Merkle ${ }^{1}$ Philipp W. Winkler ${ }^{1}$ - Stephanie Geyer ${ }^{1}$ - Elmar Herbst ${ }^{2}$ - Sepp Braun ${ }^{4,5}$. \\ Andreas B. Imhoff ${ }^{1}$ (I) . Matthias J. Feucht ${ }^{1,3}$
}

Received: 11 November 2020 / Accepted: 7 December 2020 / Published online: 23 January 2021

(c) The Author(s) 2021

\begin{abstract}
Purpose To compare post-operative physical activity and return to work after combined posterolateral corner (PLC) reconstruction (PLC-R) in anterior cruciate ligament (ACL)- or posterior cruciate ligament (PCL)-based injuries.

Methods Patients aged $>18$ years undergoing PLC-R using the Larson technique combined with either ACL or PCL reconstruction were included. Outcome was evaluated retrospectively after a minimum follow-up of 24 months using Tegner Activity Scale, Activity Rating Scale (ARS), Knee Injury and Osteoarthritis Outcome Score (KOOS), work intensity according to REFA classification, and a questionnaire about type of occupation and time to return to work.

Results A total of 32 patients (11 ACL-based injuries and 21 PCL-based injuries) were included. Mean follow-up was $56 \pm 26$ months in the ACL-based injury group and $59 \pm 24$ months in the PCL-based injury group. All patients in the ACLbased injury group and $91 \%$ of patients in the PCL-based injury group returned to sports activities. Comparing pre- and post-operative values, a significant deterioration of the Tegner Activity Scale and ARS was observed in the PCL-based injury group, whereas no significant change was observed in the ACL-based injury group. KOOS subscales were generally higher in the ACL-based injury with significant differences in the subscale sports and recreational activities. Patients with ACL-based injuries returned to work significantly earlier compared to patients with PCL-based injuries ( $11 \pm 4$ weeks vs. $21 \pm 10$ weeks, $p<0.05$ ).

Conclusion High rates of return to sports and work can be expected after combined PLC-R in both ACL- and PCL-based injuries. However, deterioration of sports ability must be expected in PCL-based injuries. ACL-based injuries led to superior patient-reported outcomes and an earlier return to work, as compared to PCL-based injuries.
\end{abstract}

Level of evidence Level IV.

Keywords Posterolateral corner · Posterior cruciate ligament $\cdot$ Anterior cruciate ligament $\cdot$ Return to sport $\cdot$ Physical activity $\cdot$ Return to work $\cdot$ Complex ligamentous knee injury $\cdot$ Multiligament knee injury

\section{Introduction}

The research was performed at the Department for Orthopedic Sports Medicine, Technical University Munich, Germany.

Andreas B. Imhoff

imhoff@tum.de

1 Department for Orthopedic Sports Medicine, Technical University Munich, Ismaninger Str. 22, 81675 Munich, Germany

2 Department of Trauma, Hand and Reconstructive Surgery, University Hospital Muenster, Westfalian-Wilhelms University Muenster, Muenster, Germany
Posterolateral corner (PLC) injuries of the knee are relatively rare and most commonly combined with concomitant ligamentous injuries $[5,7,21]$. Injuries to the PLC result in

3 Department of Orthopedics and Trauma Surgery, Medical Center, Faculty of Medicine, Albert-Ludwigs-University of Freiburg, Freiburg, Germany

4 Gelenkpunkt - Sports- and Traumasurgery Innsbruck, Innsbruck, Austria

5 Research Unit for Orthopaedic Sports Medicine and Injury Prevention, Private University for Health Sciences, Medical Informatics and Technology, Hall, Austria 
functional impairment, involving instability and pain which consecutively may accelerate the development of knee osteoarthritis [16].

Posterior cruciate ligament (PCL) tears are frequently combined with injuries to the PLC [10, 21, 30, 32, 42]. Biomechanically, isolated PCL reconstruction (PCL-R) is not able to restore native knee kinematics in combined PCLPLC injuries [13, 24, 36]. However, while many authors focused on combined PCL-PLC injuries, little is known about less common combined anterior cruciate ligament (ACL) and PLC injuries [18, 21, 37, 39]. From a biomechanical point of view, insufficiency of the PLC increases varus load on the ACL graft, which may lead to a higher failure rate after isolated ACL reconstruction (ACL-R) [20, 29, 45]. Therefore, ACL-R or PCL-R should be combined with PLC-R in combined injuries to restore nearly normal biomechanics $[6,33]$.

Satisfying functional outcomes have been reported after PLC-R combined with either ACL-R [2, 34, 43] or PCL-R $[9,11,17,25,40]$. However, these usually young and active patients have high demands concerning post-operative sports activity and work ability. These specific outcome measures are still underreported in the current literature. With our study, an improved patient education regarding post-operative sports activity, work ability, and return-to-work time following such complex ligamentous knee injuries can be achieved.

The purpose of this study was to compare post-operative sports activity and work ability between patients undergoing combined PLC-R for either ACL- or PCL-based injuries using validated patient-reported outcome scores.

The hypothesis was that PCL-based PLC injuries would result in lower return-to-sports rates and reduced work ability as compared to ACL-based injuries.

\section{Materials and methods}

This retrospective study was conducted to compare postoperative sports activity and work ability in patients undergoing combined PLC-R for either ACL- or PCL-based injuries. The study was approved by the institutional review board of the Technical University of Munich (520/17 S) and conducted according to the Declaration of Helsinki. All subjects gave their written informed consent to participate in this investigation.

For the purpose of the study, patients undergoing combined PLC-R with either ACL-R or PCL-R between 2011 and 2017 were included. Indications for PLC-R were: acute or chronic grade III PLC injury confirmed by (1) magnetic resonance imaging and (2) clinical examination (positive varus stress test at $0^{\circ}$ and $30^{\circ}$ of knee flexion and positive dial test at $30^{\circ}$ of knee flexion). Further inclusion criteria were: age $>18$ years and post-operative follow-up of at least 24 months. Exclusion criteria were: relevant comorbidities (infectious diseases, cancer, severe cardiovascular diseases), previous ligamentous reconstructions to either knee, concomitant osteotomy, medial collateral ligament reconstruction, lack of language skills, and missing consent to participate.

\section{Operative technique and post-operative rehabilitation}

In both groups, an isometric reconstruction of the PLC based on the description of Larson with a semitendinosus tendon autograft was performed [22]. Briefly, a 4-5-mm bone tunnel was created in the fibular head in an anterolateral to posteromedial direction. For the femoral tunnel, a K-wire was placed anterior and cranial to the lateral epicondyle. After isometry testing, the guidewire was overreamed with a cannulated drill according to the diameter of the graft. Subsequently, the graft was passed through the fibular tunnel and fixed with a bio-absorbable tenodesis screw (Arthrex, Naples, USA). The graft was then passed underneath the biceps tendon and iliotibial band and pulled inside the femoral tunnel. Femoral fixation was done in $30^{\circ}$ knee flexion, slight internal rotation, and valgus stress with a bio-absorbable interference screw (Arthrex, Naples, USA).

In the ACL-based injury group, an anatomic ACL-R technique with hamstring tendon autografts was performed. The femoral tunnel was drilled via an anteromedial portal according to the diameter of the graft. The tibial tunnel was placed in the centre of the tibial ACL footprint. The graft was secured at $20^{\circ}$ of flexion with an extra-cortical suspension device (ACL TightRope, Arthrex, Naples, USA) on the femoral site and with a bio-absorbable interference screw (Arthrex, Naples, USA) tibially.

In the PCL-based injury group, an anatomic single-bundle PCL-R technique (anterolateral bundle reconstruction) with a hamstring or quadriceps tendon autograft was performed. The femoral tunnel was drilled via a deep anterolateral portal according to the diameter of the graft. A guidewire was then placed in the centre of the tibial PCL footprint and overreamed according to the diameter of the graft. After graft passage, fixation was performed using bio-absorbable interference screws (Arthrex, Naples, USA).

The post-operative rehabilitation protocol in the ACLbased injury group consisted of 6 weeks of partial weight bearing on crutches. During the first two weeks, a hinged brace (Medi M4, Medi Bayreuth, Germany) with limited range of motion $(\mathrm{ROM})\left(\right.$ extension/flexion $0^{\circ} / 20^{\circ} / 90^{\circ}$ ) was used. After 2 weeks, ROM was limited to extension/flexion $0^{\circ} / 10^{\circ} / 90^{\circ}$ for 4 weeks.

The post-operative protocol in the PCL-based injury group consisted of 6 weeks of partial weight bearing on 
crutches. During the first 6 weeks, a specific brace in full extension with posterior tibial support (Medi PTS, Medi Bayreuth, Germany) was used. Only passive ROM of $0^{\circ} / 0^{\circ} / 90^{\circ}$ in prone position was allowed. After 6 weeks, a hinged brace with posterior tibial support (Medi PCL Dynamic or Medi PCL Jack, Medi Bayreuth, Germany) was used without limitations in ROM for additional 18 weeks.

Return-to-sport-specific training in both groups was allowed after 6 months and full return to contact and/or pivoting sports activities after a minimum of 9 months post-operatively.

\section{Data collection}

Medical records were reviewed to collect patient demographics and details about the medical history and surgery.

Physical activity was evaluated by Tegner Activity Scale [38], Activity rating scale [28], and a questionnaire regarding sports disciplines, sports ability, and frequency. Knee Injury and Osteoarthritis Outcome Score (KOOS) was used to evaluate self-administered knee function [35]. Time to return to work was calculated. Furthermore, work ability was evaluated by the use of questions regarding pre- and postoperative type of occupation and work intensity (according to "REFA" classification) [15].

\section{Statistical analysis}

Statistical analysis was performed using SPSS software version 25.0 (IBM-SPSS, New York, USA). Continuous variables were calculated as mean \pm standard deviation allowing one decimal. Categorical variables were reported as count and percentages allowing no decimal. Normal distribution of all data was evaluated with the Shapiro-Wilk test. The Wilcoxon signed-rank test was used to compare preoperative and post-operative nonparametric continuous variables allowing three decimals. Group comparison for continuous variables was performed with Mann-Whitney $U$ test and unpaired $t$ test, as appropriate, allowing three decimals. The chi-square test was used to compare categorical variables allowing three decimals. A $p$ value of less than 0.05 was considered to indicate statistical significance.

Post-operative sports activity was considered to be the primary outcome measure. Accordingly, the KOOS subscale sports and recreational activities were used for a priori power analysis. Assuming a standard deviation of 20 points and a mean value of 50 and 72 points for combined ACLand PCL-based PLC injuries, respectively, an effect size of 1.1 was calculated [41]. Considering the lower incidence of ACL-based PLC injuries compared to PCL-based PLC injuries, a group allocation of 1:2 was assumed. Consequently, a total sample size of 32 patients (ACL-based PLC injuries, $n=11$; PCL-based PLC injuries, $n=21$ ) was required to achieve a statistical power of 0.8 .

\section{Results}

Out of 36 patients who met the inclusion criteria, a total of 32 patients could be included for final analysis (follow-up rate, 89\%). Details of enrolment are shown in Fig. 1.

Demographic data are shown in Table 1. No significant differences with regard to demographics were observed between the two groups.

The most common cause of ACL-based injuries was sports injuries (82\%), followed by accidents during activities of daily living (18\%). The most common cause of PCLbased injuries was traffic accidents (52\%), followed by sports injuries (24\%), and accidents during activities of daily living (14\%) or work (10\%) (Fig. 2). Statistically significant more sport-related injuries were observed in the ACL-based injury group as compared to the PCL-based injury group ( $82 \%$ vs. $24 \%, p<0.001)$. On the other hand, traffic-related injuries were more frequent in the PCL-based injury group (52\% vs. $0 \%, p=0.003)$.
Fig. 1 Flow chart of patient enrolment. Concomitant surgical interventions that were excluded were: ACL + PCL and PLC reconstruction $(n=8)$, MPFL reconstruction $(n=2)$, MCL reconstruction or repair $(n=9) ; A C L$ anterior cruciate ligament, $M C L$ medial collateral ligament, $M P F L$ medial patellofemoral ligament, $P C L$ posterior cruciate ligament, $P L C$ posterolateral corner, $R$ reconstruction
55 consecutive patients underwent arthroscopic $A C L / P C L$ and $P L C$ reconstruction

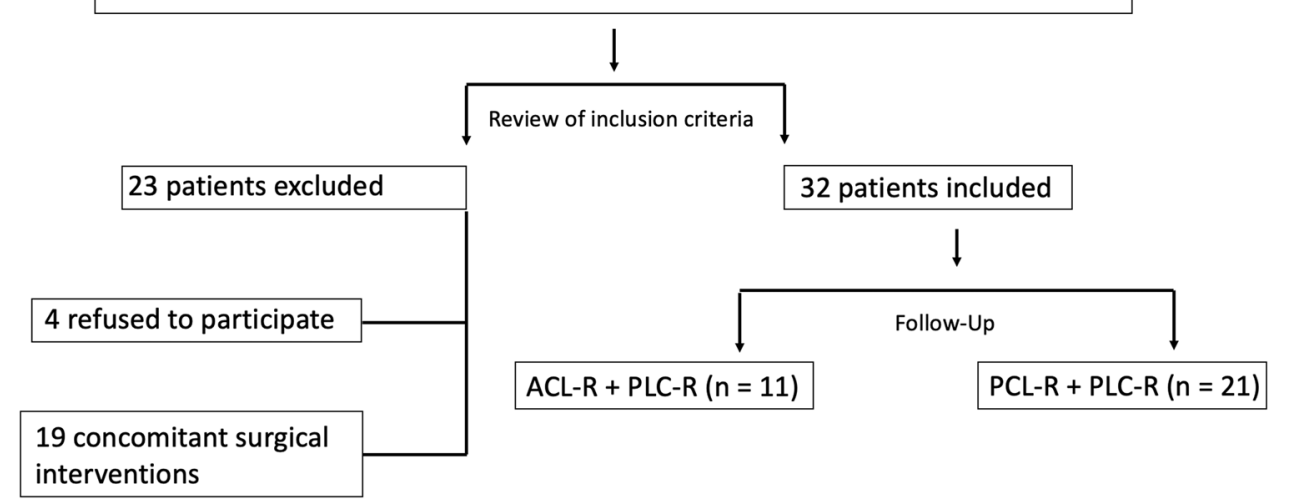


Table 1 Descriptive statistics of the demographic data and main parameters of the total study group

\begin{tabular}{llll}
\hline & ACL-based injury & PCL-based injury & $p$ value \\
\hline Number of patients, $n$ & 11 & 21 & \\
Follow-up (months) & $55.7 \pm 25.5(25-94)$ & $59.0 \pm 24.2(24-101)$ & n.s \\
Age at surgery (years) & $30.0 \pm 6.1(22-41)$ & $34.0 \pm 14.0(18-63)$ & n.s \\
Sex, $n(\%)$ & & & n.s \\
$\quad$ Male & $10(91 \%)$ & $17(81 \%)$ & \\
Female & $1(9 \%)$ & $4(19 \%)$ & n.s \\
BMI $\left(\mathrm{kg} / \mathrm{m}^{2}\right)$ & $25.2 \pm 2.3(22-29)$ & $25.5 \pm 3.9(19-32)$ & n.s \\
Time to surgery (months) & $23.3 \pm 52.0(0-174)$ & $33.2 \pm 49.4(1-181)$ & \\
Concomitant procedures, $n(\%)$ & & & \\
$\quad$ None & $9(82 \%)$ & $5(24 \%)$ & \\
Partial resection of meniscus & $2(18 \%)$ & $2(10 \%)$ & \\
$\quad$ Meniscus repair & $0(0 \%)$ & $19(90 \%)$ & \\
Post-operative complications, $n(\%)$ & & $1(5 \%)$ & \\
$\quad$ None & $11(100 \%)$ & $1(5 \%)$ & \\
Infection with Staphylococcus & $0(0 \%)$ & & \\
$\quad$ epidermidis & $0(0 \%)$ & Re-instability
\end{tabular}

Continuous variables are shown as mean \pm standard deviation (range); categorical variables are shown as percentages

$A C L$ anterior cruciate ligament, $B M I$ body mass index, n.s. not significant, $P C L$ posterior cruciate ligament, $P L C$ posterolateral corner

Groups

$A C L$-based PLC injury

PCL-based PLC injury

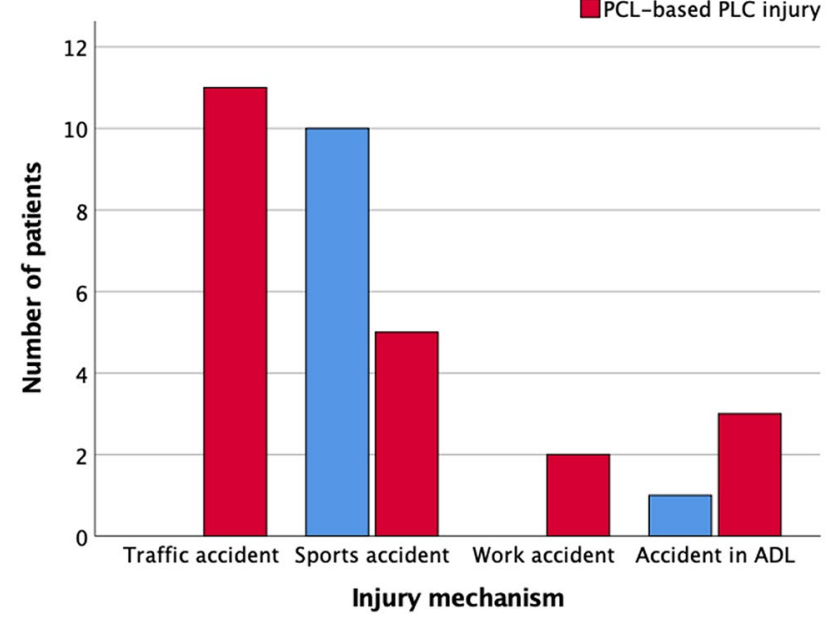

Fig. 2 PLC injury mechanism in ACL-based injuries and PCL- based injuries. Group comparison revealed a statistically significant difference with respect to the injury mechanism $(p<0.001) A D L$ activities of daily living, $P L C$ posterolateral corner, $A C L$ anterior cruciate ligament, $P C L$ posterior cruciate ligament

\section{Sports activity}

Results of sports activity are summarized in Table 2.

In the ACL-based injury group, no statistically significant difference between pre- and post-operative Tegner Activity Scale and ARS was observed (n.s.), whereas both scores decreased significantly in the follow-up period in the PCLbased injury group $(p=0.001)$. Concerning Tegner Activity Scale and ARS, no significant difference between groups could be found pre- and post-operatively (n.s.).

At the final follow-up, all subjects with an ACL-based injury and $91 \%$ of patients in the PCL-based injury group were involved in sports activities, mostly cycling and swimming (Table 3). In general, frequency of sports activities decreased from $2.0 \pm 1.5$ times per week preoperatively to $1.8 \pm 1.5$ times at the final follow-up (n.s.) in the ACL-based injury group. In the PCL-based injury group, frequency of sports activities significantly decreased from $2.6 \pm 1.3$ times per week preoperatively to $1.5 \pm 1.4$ times post-operatively $(p=0.007)$. Group comparison (ACL-based injuries vs. PCL-based injuries) showed no statistically significant difference with respect to post-operative reduction in sports activity (n.s.) (Table 2). Concerning post-operative sports performance levels, a statistically significant difference between both groups was found $(p=0.045)$, with significant lower levels in PCL-based injuries.

\section{Knee Injury and Osteoarthritis Outcome Score (KOOS)}

Post-operative KOOS subscales were generally higher in the ACL-based injuries; however, statistical significance was only reached for the KOOS subscale sports and recreational activities $(85.0 \pm 15.7$ vs. $59.3 \pm 26.1, p=0.006)$ (Table 2$)$. 
Table 2 Physical activity and clinical outcome scores of the total study group

\begin{tabular}{llll}
\hline & ACL-based injury & PCL-based injury & p value \\
\hline TEGNER post-operative & $5^{*}(2-8)$ & $4^{*}(1-8)^{\$}$ & n.s \\
TEGNER preoperative & $6^{*}(2-10)$ & $5^{*}(3-10)^{\$}$ & n.s \\
ARS post-operative & $3.0 \pm 3.5(0-12)$ & $2.1 \pm 3.8(0-13)^{\$}$ & n.s \\
ARS preoperative & $7.6 \pm 5.1(0-14)$ & $6.6 \pm 5.0(0-16)^{\$}$ & n.s \\
KOOS subscale scores & & & \\
Symptoms & $81.2 \pm 22.1(29-96)$ & $76.4 \pm 16.2(46-100)$ & n.s \\
Pain & $89.6 \pm 12.3(58-100)$ & $80.7 \pm 14.8(44-100)$ & n.s \\
ADL & $94.7 \pm 6.6(84-100)$ & $86.8 \pm 12.0(65-100)$ & n.s \\
Sports and recreational activities & $85.0 \pm 15.7(55-100)$ & $59.3 \pm 26.1(15-100)$ & $0.006^{\$}$ \\
QoL & $69.3 \pm 18.4(31-100)$ & $62.5 \pm 23.0(25-94)$ & n.s \\
Post-operative reduction in sports activity & & & \\
$\quad \begin{array}{l}\text { None, } n(\%) \\
\text { Yes, because of knee joint complaints, } n(\%)\end{array}$ & $4(36 \%)$ & $5(24 \%)$ & $13(62 \%)$ \\
$\quad$ Yes, because of other reasons (family, career, & $2(18 \%)$ & $3(14 \%)$ & \\
$\quad$ other interests), $n(\%)$ & & & \\
\hline
\end{tabular}

Continuous variables are shown as mean \pm standard deviation (range), categorical variables are shown as percentages

*Values are median; \$significant difference between pre- and post-operative TEGNER and ARS $(p=0.001)$; TEGNER Tegner Activity Scale, ARS Activity Rating Scale, KOOS Knee Injury and Osteoarthritis Outcome Score, $A D L$ activities of daily living, QoL quality of life, $n . s$. not significant
Table 3 Details of sports activity after posterolateral corner reconstruction

\begin{tabular}{llllll}
\hline Type of sport & \multicolumn{2}{l}{ ACL-based injury } & & \multicolumn{2}{c}{ PCL-based injury } \\
\cline { 2 - 3 } \cline { 6 - 6 } \cline { 5 - 6 } & Preinjury & Follow-up & & Preinjury & Follow-up \\
\hline Cycling & $10(91 \%)$ & $10(91 \%)$ & & $20(95 \%)$ & $18(86 \%)$ \\
Jogging & $7(64 \%)$ & $6(55 \%)$ & & $13(62 \%)$ & $4(19 \%)$ \\
Soccer & $8(73 \%)$ & $1(9 \%)$ & & $7(33 \%)$ & $2(10 \%)$ \\
Skiing & $2(18 \%)$ & $1(9 \%)$ & & $4(19 \%)$ & $4(19 \%)$ \\
Swimming & $8(73 \%)$ & $8(73 \%)$ & & $11(52 \%)$ & $10(48 \%)$ \\
Hiking & $5(46 \%)$ & $5(46 \%)$ & & $10(48 \%)$ & $5(24 \%)$ \\
Fitness & $3(27 \%)$ & $3(27 \%)$ & & $10(48 \%)$ & $8(38 \%)$ \\
Volleyball & $3(27 \%)$ & $2(18 \%)$ & & $1(5 \%)$ & $1(5 \%)$ \\
Table Tennis & $3(27 \%)$ & $3(27 \%)$ & & $3(14 \%)$ & $1(5 \%)$ \\
Badminton & $2(18 \%)$ & $1(9 \%)$ & & $4(19 \%)$ & $1(5 \%)$ \\
Dancing & $1(9 \%)$ & $1(9 \%)$ & & $3(14 \%)$ & $2(10 \%)$ \\
\hline
\end{tabular}

$A C L$ anterior cruciate ligament, $P C L$ posterior cruciate ligament

Values are expressed as number of patients (percentage of patients) who performed the sport activities before the injury and at follow-up. Multiple answers were possible

\section{Return to work}

The average time to return to work was significantly shorter in the ACL-based injury group as compared to the PCLbased injury group $(10.9 \pm 3.9$ weeks vs. $21.3 \pm 10.4$ weeks; $p=0.003$ ).

Preoperatively, $27 \%(n=3)$ of the subjects in the ACLbased injury group had an occupation involving heavy physical work, while $73 \%(n=8)$ worked in occupations that required little physical work. Post-operative physical workload did not change in $82 \%(n=9)$, whereas improvement or deterioration was observed in 1 patient each.

In the PCL-based injury group, 62\% $(n=13)$ had an occupation including heavy physical workload preoperatively, while $38 \%(n=8)$ patients worked in occupations that required little physical work. Post-operatively, 67\% $(n=14)$ showed no change in physical workload, whereas deterioration was observed in 24\% $(n=5)$. Group comparison showed no statistically significant difference with respect to changes in workload (n.s.).

\section{Discussion}

The most important finding of the current study was that sports activity (Tegner Activity Scale, ARS) significantly decreased post-operatively in PCL-based injuries, whereas no significant change was observed in ACL-based injuries. Furthermore, significantly more patients had to reduce the frequency of sports activities in the PCL-based injury group. Second, time to return to work was significantly longer in PCL-based injuries. Another important finding was that injury mechanisms differed significantly between ACL- and PCL-based PLC injuries. Whereas most ACL-based injuries were sports injuries, PCL-based injuries usually occurred due to traffic accidents.

While clinical outcomes after PLC-R with additional ligamentous injuries are well described $[2,4,9,11,12,17$, 
$25,34,41,43]$, the available literature mainly focused on the outcome regarding complications, re-instability, and functional scores. Only one clinical study by Wajsfisz et al. assessed the functional outcome and return to work following PLC-R with additional ACL-R or PCL-R [41]. However, there is a lack of evidence when comparing sports activity and especially return to work between ACL-based and PCLbased PLC injuries.

So far, research on return-to-sport activities has concentrated on results after isolated ACL-R or PCL-R [8, 14, $23,26,27,46]$. However, after isolated ACL-R, return-tosport activities is mostly possible with clear limitations in terms of the post-operative sports activity level [14, 26, 27]. Return to sports after isolated PCL-R was only $44 \%$ in a systematic review by Devitt et al. [8], and restrictions to postoperative activity levels [46], sport activities, and physical performance were reported [23]. These results are further strengthened by the present study on combined ligamentous injuries which also showed that patients were rarely involved in high-impact sports post-operatively (Table 3 ). In terms of patient education, this may require further preoperative expectation management of young and active patients with PLC injuries and additional rupture of the anterior or posterior cruciate ligament.

It is known that additional injuries to the PLC, such as cruciate ligament tears, have a negative impact on the clinical outcome $[4,40]$. It has been reported that post-operative functional gains after isolated PCL-R are comparable to isolated ACL-R. However, mean preoperative scores in PCL patients are lower and therefore end up at a lower final score compared to ACL patients [31]. Compared to a study after isolated ACL-R and PCL-R [31], KOOS subscales in the current cohort were higher for both groups even though the PLC was additionally reconstructed. In contrast to the results of Wajsfisz et al. following PLC-R with additional ACL-R or PCL-R [41], significant group differences in the KOOS sports and recreational activities subscale could be shown. Similar to their results, no significant group differences in other KOOS subscales were evident. Furthermore, KOOS subscales in our ACL-based injury group were comparable to the results of Cartwright et al. after combined PLC and ACL-R [4].

In the present study, a significant deterioration of Tegner Activity Scale, ARS, and frequency of sport activities could be demonstrated for the PCL-based injury group. Reasons for lower scores after combined PLC- and PCL-R are likely to be associated with heterogeneous aetiologies with higher exposures to high-impact injury mechanisms with following injuries to deep ligamentous structures that are not addressed sufficiently by PLC-R and PCL-R. In line with the literature, time to surgery in our cohort was longer in the PCL-based injury group [34]. This may explain the restrictions to postoperative return-to-sport activities and further existing knee joint complaints, since further progression of knee osteoarthritis due to ligamentous instability could have taken place in that prolonged time to surgical reconstruction [3].

Another important finding of the present study was that the return-to-work time was significantly prolonged in the PCL-based injury group with post-operative work load deterioration in $24 \%$ of patients. The long return-to-work time after PCL-R might be due to the rehabilitation protocol including strict non-weight bearing with immobilization in full extension and posterior tibial support, followed by functional bracing for up to 6 months [19]. Our results have strengthened the assumption that, compared to isolated ACL or PCL injuries [15, 44], a high return-to-work rate can be expected for patients after PLC-R with additional ACL-R or PCL-R. With our study, an improved patient education regarding return-to-work time and possible changes in work load following such complex ligamentous knee injuries can be achieved.

Various techniques have been described to treat acute or chronic PLC injuries. In the present study, the Larson technique [22] has been used to reconstruct the PLC. However, there has been a significant change in recent years towards a more anatomic technique. More recently, the LaPrade et al. [20] or the Arciero [1] technique is preferred at the authors' institution. Therefore, further research after more anatomic PLC reconstruction techniques is indicated.

Along with certain strengths, there are some limitations to this study. First, this is a retrospective study. On the other hand, there are no other studies reporting on physical activity and return to work after PLC-R with concomitant ACL-R or PCL-R. Second, the number of patients in this study is low. However, this is attributable to the low incidence of the injury patterns studied. Furthermore, the statistical power of the current study has been shown to be 0.8 . Third, only the PLC-R technique described by Larson et al. [22] was used in this study. Thus, concerning the PLC intervention, a more homogeneous cohort was achieved. Fourth, additional meniscus injuries were not excluded from this study. However, according to previous research, meniscus lesions did not affect return-to-sports activity after ACL-R [27].

The results of this study have clinical relevance when considering post-operative outcomes, including physical activity and work ability, after reconstruction of ACL- or PCL-based PLC injuries. In future, findings of this study can clinically help to improve preoperative patient counselling regarding post-operative expectations.

\section{Conclusion}

High rates of return to sports and work can be expected after combined PLC-R in both ACL- and PCL-based injuries. However, deterioration of sports ability must be expected 
in PCL-based injuries. ACL-based injuries led to superior patient-reported outcomes and an earlier return to work, as compared to PCL-based injuries.

Author contributions MJF and PML designed the study. MM, PWW, and SG collected the data. MJF and PML performed the statistical analysis and wrote the manuscript. MM, PWW, SG, EH, and SB helped to design the study, assisted with statistical analysis and data interpretation, and critically reviewed the manuscript. ABI conceived of the study, helped with data interpretation, and critically reviewed the manuscript. All authors read and approved the final manuscript.

Funding Open Access funding enabled and organized by Projekt DEAL.

\section{Compliance with ethical standards}

Conflict of interest Andreas B. Imhoff is a consultant for Arthrosurface and Medi Bayreuth and receives royalties from Arthrex and Arthrosurface. All other authors declare that they have no conflict of interest related to this study. Sepp Braun is a consultant for Arthrex.

Ethical approval Ethical approval was obtained from the Ethics Committee of the technical University Munich. The study was approved by the institutional review board of the Technical University of Munich (520/17 S). All procedures performed were in accordance with the ethical standards of the institutional and/or national research committee and with the 1964 Declaration of Helsinki and its later amendments or comparable ethical standards.

Informed consent All subjects gave their written informed consent to participate in this investigation.

Open Access This article is licensed under a Creative Commons Attribution 4.0 International License, which permits use, sharing, adaptation, distribution and reproduction in any medium or format, as long as you give appropriate credit to the original author(s) and the source, provide a link to the Creative Commons licence, and indicate if changes were made. The images or other third party material in this article are included in the article's Creative Commons licence, unless indicated otherwise in a credit line to the material. If material is not included in the article's Creative Commons licence and your intended use is not permitted by statutory regulation or exceeds the permitted use, you will need to obtain permission directly from the copyright holder. To view a copy of this licence, visit http://creativecommons.org/licenses/by/4.0/.

\section{References}

1. Arciero RA (2005) Anatomic posterolateral corner knee reconstruction. Arthroscopy 21:1147

2. Bonanzinga T, Zaffagnini S, Grassi A, Marcheggiani Muccioli GM, Neri MP, Marcacci M (2014) Management of combined anterior cruciate ligament-posterolateral corner tears: a systematic review. Am J Sports Med 42:1496-1503

3. Boynton MD, Tietjens BR (1996) Long-term followup of the untreated isolated posterior cruciate ligament-deficient knee. Am J Sports Med 24:306-310

4. Cartwright-Terry M, Yates J, Tan CK, Pengas IP, Banks JV, McNicholas MJ (2014) Medium-term (5-year) comparison of the functional outcomes of combined anterior cruciate ligament and posterolateral corner reconstruction compared with isolated anterior cruciate ligament reconstruction. Arthroscopy 30:811-817

5. Chahla J, Moatshe G, Dean CS, LaPrade RF (2016) Posterolateral corner of the knee: current concepts. Arch Bone Jt Surg 4:97-103

6. Dean RS, LaPrade RF (2020) ACL and posterolateral corner injuries. Curr Rev Musculoskelet Med 13:123-132

7. Delee JC, Riley MB, Rockwood JRCA (1983) Acute posterolateral rotatory instability of the knee. Am J Sports Med 11:199-207

8. Devitt BM, Dissanayake R, Clair J, Napier RJ, Porter TJ, Feller JA et al (2018) Isolated posterior cruciate reconstruction results in improved functional outcome but low rates of return to preinjury level of sport: a systematic review and meta-analysis. Orthop J Sports Med 6:2325967118804478

9. Fanelli GC, Edson CJ (2004) Combined posterior cruciate ligament-posterolateral reconstructions with Achilles tendon allograft and biceps femoris tendon tenodesis: 2- to 10-year follow-up. Arthroscopy 20:339-345

10. Geeslin AG, LaPrade RF (2010) Location of bone bruises and other osseous injuries associated with acute grade III isolated and combined posterolateral knee injuries. Am J Sports Med 38:2502-2508

11. Geeslin AG, LaPrade RF (2011) Outcomes of treatment of acute grade-III isolated and combined posterolateral knee injuries: a prospective case series and surgical technique. J Bone Joint Surg Am 93:1672-1683

12. Godin JA, Cinque ME, Pogorzelski J, Moatshe G, Chahla J, LaPrade RF (2017) Multiligament knee injuries in older adolescents: A 2-year minimum follow-up study. Orthop J Sports Med 5:2325967117727717

13. Harner CD, Vogrin TM, Höher J, Ma CB, Woo SL (2000) Biomechanical analysis of a posterior cruciate ligament reconstruction. Deficiency of the posterolateral structures as a cause of graft failure. Am J Sports Med 28:32-39

14. Hirschmann MT, Iranpour F, Müller W, Friederich NF (2010) Surgical treatment of complex bicruciate knee ligament injuries in elite athletes: what long-term outcome can we expect? Am J Sports Med 38:1103-1109

15. Ihle C, Ateschrang A, Albrecht D, Mueller J, Stöckle U, Schröter $S$ (2014) Occupational consequences after isolated reconstruction of the insufficient posterior cruciate ligament. BMC Res Notes 7:201

16. Kannus P (1989) Nonoperative treatment of grade II and III sprains of the lateral ligament compartment of the knee. Am J Sports Med 17:83-88

17. Kim SJ, Lee SK, Kim SH, Kim SH, Jung M (2013) Clinical outcomes for reconstruction of the posterolateral corner and posterior cruciate ligament in injuries with mild grade 2 or less posterior translation: comparison with isolated posterolateral corner reconstruction. Am J Sports Med 41:1613-1620

18. Kinsella SD, Rider SM, Fury MS, Tepolt FA, Ecklund K, Kocher MS (2019) Concomitant posterolateral corner injuries in skeletally immature patients with acute anterior cruciate ligament injuries. J Pediatr Orthop. https://doi.org/10.1097/bpo.0000000000001450

19. Klose M, Forkel P, Imhoff A (2018) Rehabilitation after reconstruction of the posterior cruciate ligament. MMW Fortschr Med 160:69-71

20. LaPrade RF, Johansen S, Wentorf FA, Engebretsen L, Esterberg JL, Tso A (2004) An analysis of an anatomical posterolateral knee reconstruction: an in vitro biomechanical study and development of a surgical technique. Am J Sports Med 32:1405-1414

21. LaPrade RF, Wentorf FA, Fritts H, Gundry C, Hightower CD (2007) A prospective magnetic resonance imaging study of the incidence of posterolateral and multiple ligament injuries in acute knee injuries presenting with a hemarthrosis. Arthroscopy 23:1341-1347 
22. Larson RV (2001) Isometry of the lateral collateral and popliteofibular ligaments and techniques for reconstruction using a free semitendinosus tendon graft. Oper Techn Sport Med 9:84-90

23. Lee DW, Kim JG, Yang SJ, Cho SI (2019) Return to sports and clinical outcomes after arthroscopic anatomic posterior cruciate ligament reconstruction with remnant preservation. Arthroscopy 35:2658-2668.e2651

24. Lee DY, Park YJ, Kim DH, Kim HJ, Nam DC, Park JS et al (2018) The role of isolated posterior cruciate ligament reconstruction in knees with combined posterior cruciate ligament and posterolateral complex injury. Knee Surg Sports Traumatol Arthrosc 26:2669-2678

25. Lind M, Nielsen TG, Behrndtz K (2018) Both isolated and multi-ligament posterior cruciate ligament reconstruction results in improved subjective outcome: results from the Danish Knee Ligament Reconstruction Registry. Knee Surg Sports Traumatol Arthrosc 26:1190-1196

26. Lindanger L, Strand T, Mølster AO, Solheim E, Inderhaug E (2019) Return to play and long-term participation in pivoting sports after anterior cruciate ligament reconstruction. Am J Sports Med 47:3339-3346

27. Mardani-Kivi M, Azari Z, Hasannejad F (2020) Return to sport activity after anterior cruciate ligament reconstruction: A 6-10 years follow-up. J Clin Orthop Trauma 11:S319-s325

28. Marx RG, Stump TJ, Jones EC, Wickiewicz TL, Warren RF (2001) Development and evaluation of an activity rating scale for disorders of the knee. Am J Sports Med 29:213-218

29. Mehl J, Otto A, Kia C, Murphy M, Obopilwe E, Imhoff FB et al (2019) Osseous valgus alignment and posteromedial ligament complex deficiency lead to increased ACL graft forces. Knee Surg Sports Traumatol Arthrosc 28:1-11

30. Owesen C, Sandven-Thrane S, Lind M, Forssblad M, Granan LP, Årøen A (2017) Epidemiology of surgically treated posterior cruciate ligament injuries in Scandinavia. Knee Surg Sports Traumatol Arthrosc 25:2384-2391

31. Owesen C, Sivertsen EA, Engebretsen L, Granan LP, Årøen A (2015) Patients with isolated PCL injuries improve from surgery as much as patients with ACL injuries after 2 years. Orthop J Sports Med 3:2325967115599539

32. Pacheco RJ, Ayre CA, Bollen SR (2011) Posterolateral corner injuries of the knee: a serious injury commonly missed. J Bone Joint Surg Br 93:194-197

33. Petrillo S, Volpi P, Papalia R, Maffulli N, Denaro V (2017) Management of combined injuries of the posterior cruciate ligament and posterolateral corner of the knee: a systematic review. Br Med Bull 123:47-57

34. Rochecongar G, Plaweski S, Azar M, Demey G, Arndt J, Louis ML et al (2014) Management of combined anterior or posterior cruciate ligament and posterolateral corner injuries: a systematic review. Orthop Traumatol Surg Res 100:S371-378
35. Roos EM, Roos HP, Lohmander LS, Ekdahl C, Beynnon BD (1998) Knee injury and osteoarthritis outcome score (KOOS)development of a self-administered outcome measure. J Orthop Sports Phys Ther 28:88-96

36. Sekiya JK, Whiddon DR, Zehms CT, Miller MD (2008) A clinically relevant assessment of posterior cruciate ligament and posterolateral corner injuries. Evaluation of isolated and combined deficiency. J Bone Joint Surg Am 90:1621-1627

37. Shaw KA, Dunoski BS, Mardis NJ, Pacicca DM (2016) Combined posterolateral corner and acute anterior cruciate ligament injuries in an adolescent cohort: a magnetic resonance imaging analysis. Int Orthop 40:555-560

38. Tegner Y, Lysholm J (1985) Rating systems in the evaluation of knee ligament injuries. Clin Orthop Relat Res 198:43-49

39. Temponi EF, de Carvalho Júnior LH, Saithna A, Thaunat M, Sonnery-Cottet B (2017) Incidence and MRI characterization of the spectrum of posterolateral corner injuries occurring in association with ACL rupture. Skeletal Radiol 46:1063-1070

40. van der Wal WA, Heesterbeek PJ, van Tienen TG, Busch VJ, van Ochten JH, Wymenga AB (2016) Anatomical reconstruction of posterolateral corner and combined injuries of the knee. Knee Surg Sports Traumatol Arthrosc 24:221-228

41. Wajsfisz A, Bajard X, Plaweski S, Djian P, Demey G, Limozin R et al (2014) Surgical management of combined anterior or posterior cruciate ligament and posterolateral corner tears: for what functional results? Orthop Traumatol Surg Res 100:S379-383

42. Wang D, Berger N, Cohen JR, Lord EL, Wang JC, Hame SL (2015) Surgical treatment of posterior cruciate ligament insufficiency in the United States. Orthopedics 38:e281-286

43. Westermann RW, Marx RG, Spindler KP, Huston LJ, Amendola A, Andrish JT et al (2019) No difference between posterolateral corner repair and reconstruction with concurrent ACL surgery: results from a prospective multicenter cohort. Orthop J Sports Med 7:2325967119861062

44. Wexler G, Bach BR Jr, Bush-Joseph CA, Smink D, Ferrari JD, Bojchuk J (2000) Outcomes of anterior cruciate ligament reconstruction in patients with Workers' Compensation claims. Arthroscopy $16: 49-58$

45. Zantop T, Schumacher T, Diermann N, Schanz S, Raschke MJ, Petersen W (2007) Anterolateral rotational knee instability: role of posterolateral structures. Winner of the AGA-DonJoy Award 2006. Arch Orthop Trauma Surg 127:743-752

46. Zayni R, Hager JP, Archbold P, Fournier Y, Quelard B, Chambat P et al (2011) Activity level recovery after arthroscopic PCL reconstruction: a series of 21 patients with a mean follow-up of 29 months. Knee 18:392-395

Publisher's Note Springer Nature remains neutral with regard to jurisdictional claims in published maps and institutional affiliations. 